\title{
The Regulation of Colicin Synthesis and Colicin Factor Transfer in Escherichia coli $\mathrm{K} 12$
}

\author{
BY MARILYN MONK AND R. C. CLOWES \\ Medical Research Council, Microbial Genetics Research Unit, \\ Hammersmith Hospital, London, W.12
}

(Received 20 March 1964)

\begin{abstract}
SUMMARY
Cells of Escherichia coli, newly infected with the colicin I factor (colI), showed an enhanced efficiency of transfer of this factor (HFC), and were also more likely to undergo lethal colicin synthesis, than were stably colicinogenic cells. Up to $20 \%$ of the cells of stably colI ${ }^{+}$strains were induced to produce colicin by ultraviolet irradiation, and from such irradiated cultures transfer of the colI factor occurred more efficiently. To account for these results, it is proposed that the colI factor exists as an autonomous non-chromosomal genetic element which sets up its own system of self-regulation within cells of stably colicinogenic strains.
\end{abstract}

\section{INTRODUCTION}

Colicin production in stably colI ${ }^{+}$strains of Salmonella typhimurium was demonstrated by Ozeki, Stocker \& de Margerie (1959) who observed the release of colicin from single bacteria as minute clearings termed 'lacunae' in a lawn of colicin-sensitive cells. An enhancement in the number of these 'lacunae' following ultraviolet (u.v.) irradiation of colicinogenic strains was shown by Ozeki (1960) and ascribed to the induction of lethal colicin synthesis in an increased number of cells of a $\mathrm{col}^{+}$ population. In Salmonella typhimurium, cells newly infected with the colI factor are highly efficient in the further transfer of this factor (Stocker, Smith \& Ozeki, 1963). It was suggested (Smith \& Stocker, 1962; Stocker et al. 1963) that in stably colicinogenic cells, the colI factor exists chromosomally, but in a small minority of cells it may take up a cytoplasmic state, in which event it becomes capable of infectious transfer at low frequency (low-frequency colicinogeny transfer, LFC). Newly transferred col factors are suggested to persist for some time in this cytoplasmic state, during which time the efficiency of further colI transfer remains high, giving rise to a system of high-frequency colicinogeny transfer (HFC) before the proposed stable chromosomal condition is re-established. We have previously shown the existence of high-frequency colicinogeny transfer (HFC) in Escherichia coli $\mathrm{K} 12$ strains newly infected with colI (Monk \& Clowes, 1964). In the present paper we report the incidence of colicin-producing cells ('lacunae') in both LFC and HFC cultures of $E$. coli $\mathrm{x} 12$, together with related studies on the induction of lacunae and colI transfer following u.v.-irradiation of stably colI ${ }^{+} E$. coli $\mathrm{x} 12$ cultures. An alternative hypothesis to account for $\mathrm{HFC}$ and colicin induction based on a system of self-regulation of non-chromosomal colI factors is proposed. 


\section{METHODS}

Media and bacterial strains. Those used in this study are described in Monk \& Clowes (1964). To avoid re-adsorption of colicin, all the colI ${ }^{+}$strains used were also colI $-r$. In addition, derivatives of a non-lysogenic $\left(\lambda^{-}\right)$strain, w 1655 (Lederberg \& Lederberg, 1953) were used in u.v.-irradiation experiments to prevent lysis and death arising from induction of the $\lambda$ prophage. Some w 1655 derivatives were isolated from a strain cured of $F$ by the method of Hirota (1960) by using acridine orange. One of these strains (59), an $\mathrm{F}^{-}$-derivative of $\mathrm{w} \mathbf{1 6 5 5}$, made resistant to colicin I (colI-r) and colicinogenic $\left(\right.$ coll $\left.I^{+}\right)$, was used in these studies.

Assay of colicin production by single cells (lacunae). To $2.5 \mathrm{ml}$. soft agar, seeded with $0.1 \mathrm{ml}$. of an overnight col-s str-r indicator strain, was added an inoculum of $10^{7}$ cells of a colI ${ }^{+}$str-s strain, and the agar layered over a streptomycin nutrient agar plate. After incubation for 5-6 hr, small clearings (lacunae) in the confluent indicator lawn were visible. Alternatively using a colI $^{+}$strain which was $s t r-r$, several drops of chloroform were added to the broth culture, which was then incubated for $10 \mathrm{~min}$. before adding diluted samples to the soft agar indicator mixture.

Ultraviolet (u.v.)-irradiation was achieved using a Hanovia 15 W. 'germicidal' lamp emitting $95 \%$ of its radiation at $2537 \AA$. Cells were irradiated at $50 \mathrm{~cm}$. from the lamp, the intensity at this distance being $5 \mathrm{ergs} / \mathrm{mm}^{2} / \mathrm{sec}$. Logarithmic-phase colI $I^{+}$bacteria, grown in nutrient broth, were centrifuged at low speed (3000 rev.) min.), and the supernatant fluid replaced with phosphate buffer $(0.5 \mathrm{M} ; \mathrm{pH} 7.5)$ to produce a cell concentration of 1 to $2 \times 10^{8} / \mathrm{ml}$. Five $\mathrm{ml}$. samples were u.v. irradiated in $9 \mathrm{~cm}$. Petri dishes for the desired time with gentle agitation. The irradiated cell suspension was then rapidly transferred to an equal volume of prewarmed, double-strength nutrient broth and incubated with aeration in the dark.

Other colicin techniques were as described previously.

\section{RESULTS}

\section{Lacunae production in $\mathrm{K} 12 \mathrm{colI}^{+}$strains}

The small clearings (lacunae) produced by $c o l I^{+}$str-s Salmonella cells in a confluent lawn of colI $^{-}$str-r indicator bacteria on a streptomycin overlay plate were ascribed by Ozeki et al. (1959) to the release of colicin by single bacteria. Lacunae produced by Escherichia coli $\mathrm{K} 12$ colI $^{+}$strains form just visible clearings in the indicator lawn and are difficult to score with precision, there being considerable variation in their size and clarity. Triplicate or quadruplicate platings were independently scored on at least two occasions, and differences were only regarded as significant when they exceeded tenfold. The frequency of lacunae observed in stably colI ${ }^{+}$cultures is generally about $1 / 10^{5}$ cells (a variation from about 3 in $10^{5}$ to 3 in $10^{6}$ cells). These clearings were confirmed as lacunae since they were not produced with cell-free filtrates, and they occurred with a frequency directly proportional to the numbers of cells plated. Moreover, they were not transferred by subculture, and were thus non-infective, and they were not detected when a colI- $r$ strain was used as an indicator, nor when the col-s indicator was plated in the absence of a coll ${ }^{+}$inoculum. 


\section{Induction of colicin I synthesis by ultraviolet-irradiation}

Although induction of colicin E 1, E 2 and B by u.v.-irradiation has been reported in Salmonella typhimurium by Ozeki (1960), induction of colicin I was not achieved. A colI $^{+}$colI-r str-s strain of Escherichia coli $\mathrm{k} 12$, non-lysogenic for phage $\lambda$, was irradiated for various times and assayed for lacunae production after $100 \mathrm{~min}$. of post-irradiation incubation. As the dose of u.v.-radiation was increased, the numbers of lacunae were increased, reaching a plateau after irradiation for $60 \mathrm{sec}$. when about $20 \%$ of the cells formed lacunae. The time of $60 \mathrm{sec}$. was used in further experiments in which the number of lacunae arising after various times of postirradiation incubation were assayed; the results are shown in Fig. 1. At this dose,

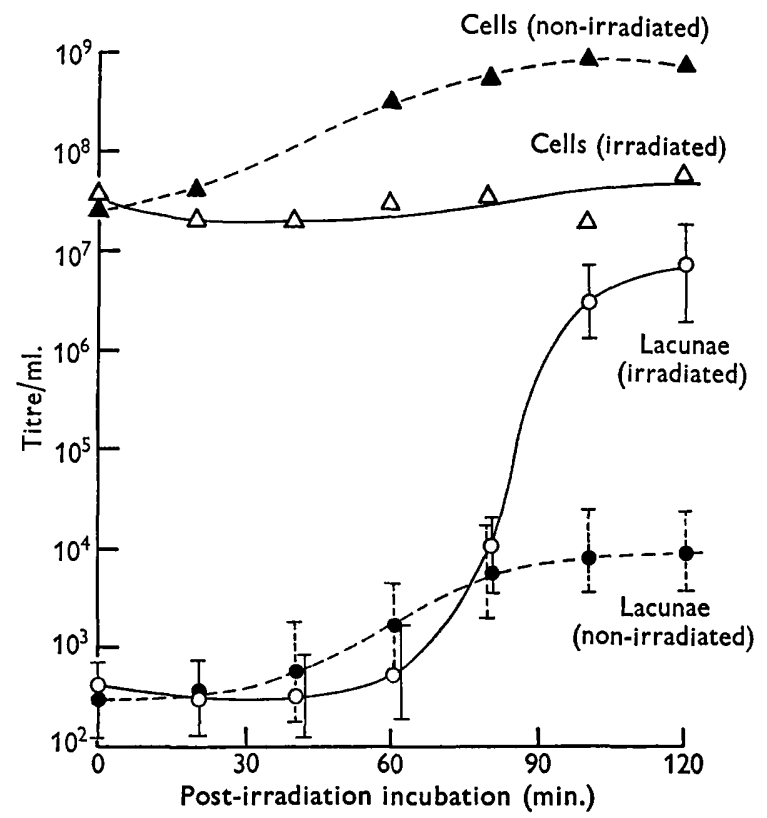

Fig. 1. Viable count and lacunae of a colI $I^{+}$culture after u.v.-irradiation. A log.-phase culture of colI $I^{+}$colI $-r$ str-s $\lambda^{-}$bacteria (strain 59) was centrifuged and resuspended at $10^{8}$ cells $/ \mathrm{ml}$. in buffer. A $5 \mathrm{ml}$. sample was removed to $5 \mathrm{ml}$. double-strength nutrient broth to serve as a control unirradiated culture. A further $5 \mathrm{ml}$. was then u.v.-irradiated for 60 sec. at $50 \mathrm{~cm}$. from the u.v. source, and then similarly diluted into $5 \mathrm{ml}$. doublestrength nutrient broth. Both samples were then incubated at $37^{\circ}$ with aeration and at various times further samples were removed and assayed on nutrient agar for viable count, and streptomycin nutrient agar with a col-s str-r indicator for the numbers of lacunae. $\Delta-\cdots \Delta$, Viable count of non-irradiated culture: $\triangle \longrightarrow \Delta$, viable count of u.v.-irradiated culture: $--\infty$, lacunae in non-irradiated culture: $0-0$, lacunae in u.v.-irradiated culture.

the irradiated cells did not show any marked decrease in viable count, although cell-division was considerably delayed. The number of lacunae remained constant at the uninduced value until about $60 \mathrm{~min}$. after irradiation, when a sharp increase occurred, reaching a maximum at $120 \mathrm{~min}$. of about $10^{7}$ lacunae $/ \mathrm{ml}$., and corresponding to an induction of about $20 \%$ of the total number of irradiated cells. At this time the amount of free colicin in the cell-free filtrate showed an increase of about 30-fold. 


\section{Enhanced transfer of colI from irradiated colI ${ }^{+}$cultures}

A culture of a stably colI ${ }^{+}$strain was u.v.-irradiated for various periods under the conditions optimal for induction, and samples of the irradiated culture mixed with equal numbers of log.-phase colI-r str-r recipient cells in double-strength broth, and re-incubated for $2 \mathrm{hr}$. The mixed culture was then diluted and the recipient cells assayed for acquisition of colI; the results are shown in Table 1. After irradiation for 10, 20 or 30 sec., a small but reproducible increase in colI transfer resulted.

\section{Table 1. Relative increase in colI transfer after u.v.-irradiation}

Log.-phase cultures of strain 59 were centrifuged and resuspended in buffer at $10^{8}$ cells $/ \mathrm{ml}$. Samples $(5 \mathrm{ml}$.) were u.v.-irradiated for the times shown, then $1 \mathrm{ml}$. samples mixed with $1 \mathrm{ml}$. log.-phase cells of a culture of 58-161 $\lambda+\mathbf{F}^{-}$colI-r str-r (550) in doublestrength nutrient broth at $10^{8}$ cells $/ \mathrm{ml}$. Mixtures were incubated for $2 \mathrm{hr}$ and $s t r-r$ cells selected and tested for $\mathrm{colI}^{+}$. Three hundred to 1000 clones were scored at each time and expressed relative to the numbers of $\mathrm{colI}^{+}$found after contact with unirradiated colonies as 100. The absolute value ranged from 5 to $20 \%$ transfer.

Time of u.v.-irradiation (sec.)

\begin{tabular}{rcccccr} 
Exp. & \multicolumn{1}{c}{$\begin{array}{c}20 \\
\text { Relative no. of colI } \text { transfer }\end{array}$} \\
& $\overbrace{100}$ & 180 & 230 & 220 & 200 & 170 \\
2 & 100 & - & 180 & - & 160 & 110 \\
3 & 100 & 310 & 360 & - & 110 & 50 \\
4 & 100 & 180 & 220 & 210 & 150 & 110 \\
5 & 100 & 140 & 150 & 130 & 100 & 80 \\
6 & 100 & 200 & 200 & 170 & 120 & 100 \\
7 & 100 & 150 & 150 & 180 & 150 & 100
\end{tabular}

This increase was from 1.3- to $\mathbf{3 \cdot 6 - f o l d , ~ c a l c u l a t e d ~ o n ~ t h e ~ n u m b e r s ~ o f ~} \mathrm{colI}^{+}$input cells. However, if the increase in infectivity is assumed to occur at the same time as the onset of induction, namely about $90 \mathrm{~min}$., the non-irradiated control cells have multiplied by a factor of about 20 . The relative transfer per viable cell might therefore be enhanced by as much as 30- to 70-fold as a result of u.v.-irradiation.

\section{Enhanced lethal colicin synthesis in HFC cultures}

When incubated overnight preparations of HFC were diluted 1/20 in broth to prepare standard HFC 'donor mixtures', it was found $15 \mathrm{~min}$. later that the numbers of 'intermediate' cells which were recovered as viable colonies had decreased to about $50 \%$. Similarly, when a coll-r recipient strain was mixed with such a HFC donor mixture, only $50-70 \%$ of the recipient cells survived as colonyformers when plated $15 \mathrm{~min}$. after mixing. Since both populations which showed this decrease were non-adsorbing for colicin I, the observed lethal effects appear to be due to a transfer of the colI factor, rather than to any direct colicin action. The number of lacunae-forming cells and free colicin were therefore determined in a HFC donor mixture and compared with similar assays in a LFC donor culture; the results are shown in Table 2. It can be seen that under HFC conditions the numbers of lacunae increased more than 100-fold, with an accompanying 16-fold increase in the cell-free colicin. 


\section{Table 2. Lethal colicin synthesis in HFC and LFC cultures}

HFC preparation: $5 \times 10^{4}$ cells of donor colI ${ }^{+} s t r-s(519)$, and $10^{6}$ cells of intermediate colI-r str-s (547) were mixedly inoculated into $5 \mathrm{ml}$. nutrient broth, incubated overnight, diluted 1 in 20 and then incubated for $90 \mathrm{~min}$. with aeration. For the control, the donor strain was mixed with the strain colI ${ }^{+}$coll-r str-s (559) and cultured in the same way. Samples were removed and (i) diluted! and plated with colI-s str-r indicator in $\mathbf{2 . 5}$ ml. soft agar on streptomycin nutrient agar for numbers of lacunae: (ii) centrifuged, and doubling dilutions of supernatant fluid spotted on a streptomycin nutrient agar indicator plate for colicin titre: (iii) mixed with log.-phase recipient colI- $r$ str-r cells (550) at a ratio of $1: 1$ and incubated for $1 \mathrm{hr}$. The proportion of coll $\mathrm{I}^{+}$recipient cells was determined by triple overlay on streptomycin nutrient agar.

\begin{tabular}{|c|c|c|c|c|}
\hline System & Viable cells $/ \mathrm{ml}$. & Lacunae $/ \mathrm{ml}$. & $\begin{array}{c}\text { Limiting colicin } \\
\text { titre }\end{array}$ & $\begin{array}{c}\text { Transfer colI } \\
\text { to recipient }(\%)\end{array}$ \\
\hline $\operatorname{colI} I^{+}(\mathrm{HFC})$ & $10^{8}$ & $5 \times 10^{5}$ & $1 / 16$ & 98 \\
\hline tably $\operatorname{colI}^{+}$ & $2 \times 10^{8}$ & $3 \times 10^{3}$ & 1 & $4 \cdot 2$ \\
\hline
\end{tabular}

\section{DISCUSSION}

In Escherichia coli, cells newly infected with colI become highly efficient donors of this factor and transmission to a non-colicinogenic recipient is increased from about $1 \%$ (LFC) to about $90 \%$ (HFC) within the first hour (Monk \& Clowes, 1964). The duration of this enhanced infectivity is about four to six generations, similar to the HFC condition first described in Salmonella typhimurium by Stocker et al. (1963). It has also been shown that an increase in the infectivity of a colI ${ }^{+}$culture is accompanied by an increase in the numbers of colI ${ }^{+}$cells which are able to form unions when mixed with non-colicinogenic recipient organisms. These unions may be observed by mixing colI ${ }^{+}$cells, which have been stained by the uptake of a tetrazolium dye, with unstained $\mathrm{col}^{-}$cells, whereupon pairing of $\mathrm{col}^{+}$and $\mathrm{col}^{-}$cells, or more frequently clumps including a number of both types of cells, may be seen. Such pairing or clumping is not seen in cultures of either strain independently. Stably $\mathrm{colI}^{+}$cultures of $\boldsymbol{E}$. coli, which can transfer colI to about 7-10\% of recipient cells in $2 \mathrm{hr}$, are able to form a small proportion of cell clumps (about $5 \%$ ), whereas in HFC $\boldsymbol{E}$. coli mixtures, a much higher proportion of the cells (50-100\%) are seen in clumps (Monk \& Clowes, 1964). S. typhimurium HFC cultures (transmitting $50 \%$ colI in $1 \mathrm{hr}$ ) also form clumps, but no pairing or association of cells is seen under LFC conditions, when the transfer in the first $2 \mathrm{hr}$ is less than $0.01 \%$ (Ozeki, Stocker $\&$ Smith, 1962). Increased infectivity would thus seem to be due, in part at least, to an increase in the numbers of $\mathrm{colI}^{+}$cells which can form stable contacts with $\mathrm{colI} \mathbf{I}^{-}$ cells. These contacts are likely to result from an increase in the numbers of cells which form a specific col-directed conjugating cell surface (possibly a col-conjugating antigen similar to that produced by the presence of the fertility factor $F$ ) or an increase in the amounts of such conjugating antigen produced by most cells. Increased colI infectivity might also be due to an increase in the numbers of colI factors per cell, increasing the probability of colI transfer per contact. Recent experiments by Dr H. Ozeki (personal communication) on the 'suicide' of ${ }^{32} \mathbf{P}$ labelled $c o l$ factors after transfer indicate that there is a rapid multiplication of these factors within the newly infected cells.

In a previous publication (Monk \& Clowes, 1964) we concluded that colI, like other colicin factors such as colE1 (see Clowes, 1964), appears to be transferred 
extra-chromosomally, and no evidence was found to suggest a chromosomal location. The multiplication and activity of a stable cytoplasmic element or plasmid (Lederberg, 1952), which is also a potentially lethal agent, yet whose lethality is seldom expressed, must be regulated in some way. Such regulation in the case of non-chromosomal elements is likely to be self-imposed and may, by analogy with other regulated and potentially lethal systems such as temperate phage, be due to the presence of a cytoplasmic repressor. This picture of the colI factor as a selfregulating plasmid, also independently suggested by Clark \& Adelberg (1962), appears to be more compatible with the data shown in the present paper than the alternative model proposed by Smith \& Stocker (1962).

In stable $\mathrm{col}^{+}$strains, the activities of the colI factor (which includes lethal synthesis of colicin, and the ability to form a conjugating cell surface) would be repressed in most cells. In a small proportion of colI ${ }^{+}$cells, this repression breaks down and lethal synthesis results. In other cells, de-repression might lead to an enhancement of non-lethal activities, such as the synthesis of the col-directed surface 'antigen' (and possibly of the numbers of col factors existing in the cell), which would allow these cells to conjugate and transfer col factors, or in other words to become 'competent donors' of colI, either without lethal colicin synthesis, or before lethality prevented transfer. This repression would appear to be less stringent in Escherichia coli than in Salmonella typhimurium, resulting in about a hundred-fold greater number both of competent donors and of cells which proceed to lethal synthesis. (In E. coli, 'competent donors' are about $1 \%$ and lethal synthesis is observed in about 1 in $10^{5}$ cells, whereas in $S$. typhimurium, competent donors are about $0.02 \%$ and lethal synthesis is found in less than 1 in $10^{7}$ cells; Ozeki et al. 1962).

Transfer of the colI factor in the absence of its repressor would lead to a period of de-repression in newly infected cells. Such cells would themselves then have an increased probability of colI transfer, which could account for the epidemic spread of colI, giving rise to HFC donor mixtures under the appropriate conditions. De-repression in such newly infected cells would also be likely to have lethal consequences. This lethality is demonstrated when overnight HFC mixtures are subc iltured, when extensive colI transfer to previously non-colicinogenic (intermedi.ate) cells is occurring. This loss of viable cells is paralleled by the high incidence of lacunae-producing cells in such cultures (Table 2). When HFC donor mixtures are mixed with recipients to which colI is transferred to $80 \%$ of the survivors after $30 \mathrm{~min}$., this is also accompanied by lethal effects in the recipients, and 50-70\% do not survive $15 \mathrm{~min}$. after mixing.

Such lethal effects following infection with colI are parallel to those subsequent to infection with temperate phage. Here infection may result in either lethal synthesis of phage, with consequent death of the cell (productive infection) or the establishment of a stably-lysogenic cell (reductive infection, Lwoff, 1953). Although in some instances, particularly the well documented phage $\lambda$ system (Jacob, 1960), such lysogeny can be shown to be due to the integration of the phage genome as a prophage at a particular site on the bacterial genome, in other systems the phage genome may persist for some time in the cytoplasm without lethal effects (Luria, Fraser, Adams \& Burrous, 1958). Moreover, even in the case of those phages in which prophage integration occurs, mutants may be isolated, such as phage $\lambda \mathrm{b2}$, 
which can no longer integrate, but retain the ability to set up an immune 'abortive' lysogenic state (Zichichi \& Kellenberger, 1963). With other phages (particularly those capable of 'generalized' transduction, such as P1 or 363), it has not so far been possible to establish a chromosomal location (Jacob \& Wollman, 1958), and they too may exist as plasmids, capable of establishing an immune, stably-lysogenic state without integration.

The destruction of a cytoplasmic repressor has been suggested to account for induction of prophage to lethal synthesis following u.v.-irradiation of a lysogenic strain (Jacob \& Monod, 1961). In colI $^{+}$cultures of Escherichia coli, u.v.-irradiation also results in greater than a thousand-fold increase in the numbers of cells which proceed to lethal synthesis (Fig. 1). Such induction is accompanied by a small but reproducible increase in the infectivity of colI, both effects being ascribed to the destruction of a regulation system. Similar effects on enhanced transfer after u.v.irradiation of strains which harbour RTF have been suggested (Watanabe \& Fukasawa, 1961) to be due to a displacement of the RTF element from the chromosomal to the cytoplasmic state. De-repression of an autonomous plasmid is an alternative hypothesis that may be equally valid in this system.

Some of this work has been submitted by one of us (M.M.) in partial fulfilment of the requirements for the Degree of Doctor of Philosophy in the University of London, who wishes to acknowledge the receipt of a Canteens Services Trust Fund (Australia) Scholarship and the generous use of facilities extended by Dr W. Hayes, F.R.S., and the Medical Research Council.

\section{REFERENCES}

Clark, A. J. \& Adelberg, E. A. (1962). Bacterial conjugation. A. Rev. Microbiol. 16, 289.

Clowes, R. C. (1964). 'Transfert génétique des facteurs colicinogènes. Annls Inst. Pasteur, Paris (in the Press).

Hirota, Y. (1960). The effect of acridine dyes on mating type factors in Escherichia coli к-12. Proc. nat. Acad. Sci., Wash. 46, 57.

$\mathrm{J}_{\mathrm{ACOB}}$, F. (1960). Genetic control of viral functions. Harvey Lect. 1958-59.

$\mathrm{J}_{\mathrm{ACOB}}, \mathrm{F}$. \& MonoD, J. (1961). Genetic regulatory mechanisms in the synthesis of proteins. J. mol. Biol. 3, 318.

$\mathrm{J}_{\mathrm{ACOB}}, \mathrm{F}$. \& Wollman, E. L. (1958). The relationship between the prophage and the bacterial chromosome in lysogenic bacteria. In Recent Progress in Microbiology, p. 15. Symposia at VII Int. Cong. Microbiol. Stockholm: Almqvist and Wiksell.

Lederberg, J. (1952). Cell genetics and hereditary symbiosis. Physiol. Rev. 32, 403.

LeDERBERG, E. M. \& LEDERBERG, J. (1953). Genetic studies on lysogenicity in interstrain crosses in $\boldsymbol{E}$. coli. Genetics, $\mathbf{3 8 ,} \mathbf{5 1}$.

Luria, S. E., Fraser, D. K., Adams, J. N. \& Burrous, J. W. (1958). Lysogenisation, transduction and genetic recombination in bacteria. Cold Spring Harb. Symp. quant. Biol. 23, 71.

LwoFf, A. (1953). Lysogeny. Bact. Rev. 17, 269.

Monk, M. \& Clowes, R. C. (1964). Transfer of the colicin I factor in Escherichia coli $\mathbf{k 1 2}$ and its interaction with the $F$ fertility factor. J. gen. Microbiol. 36, 365.

Ozenr, H. (1960). Colicinogeny in Salmonella: Genetic and other Studies. Ph.D. Thesis, Univ. London.

Ozeki, H., Stocker, B. A.D. \& de Margerie, H. (1959). Production of colicine by single bacteria. Nature, Lond. 184, 337. 
Ozeki, H., Stocker, B. A. D. \& Smith, S. M. (1962). Transmission of colicinogeny between strains of Salmonella typhimurium grown together. J. gen. Microbiol. 28, 671 .

Smith, S. \& Stocker, B. A. D. (1962). Colicinogeny and recombination. Br. med. Bull. $18,46$.

Stocker, B. A. D., Sмrth, S. M. \& Ozeki, H. (1963). High infectivity of Salmonella typhimurium newly infected by the colI factor. J. gen. Microbiol. 30, 201.

Watanabe, T. \& Fukasawa, T. (1961). Episome-mediated transfer of drug resistance in Enterobacteriaceae. II. Elimination of resistance factors with acridines. J. Bact. 81, 679.

Zichichi, M. L. \& Kellenberger, G. (1963). Two distinct functions in the lysogenisation process: the repression of phage multiplication and the incorporation of the prophage in the bacterial genome. Virology, 19, 450. 